\title{
TYPES OF ANGIOPATHY IN EXPERIMENTAL AUTOIMMUNE DISEASE IN RATS
}

\author{
Yelizaveta Iegudina \\ Department of Propedeutic of Internal Medicine \\ SE "Dnepropetrovsk Medical Academy of Health Ministry of Ukraine" \\ 9 Dzerzhinsky str., Dnipro, Ukraine, 49044 \\ elizavetaegudina@gmail.com \\ Pavel Yakovlenko \\ Department of Internal Medicine № 1 \\ M. Gorky Donetsk National Medical University \\ 27 Privokzalna str., Liman, Ukraine 84404 \\ Oleg Syniachenko \\ Department of Internal Medicine № 1 \\ M. Gorky Donetsk National Medical University \\ 27 Privokzalna str., Liman, Ukraine, 84404 \\ Viktoriya Mikuksts \\ Department of Internal Medicine № 1 \\ M. Gorky Donetsk National Medical University \\ 27 Privokzalna str., Liman, Ukraine, 84404
}

\begin{abstract}
The purpose and objectives of this work were to study the nature of the heart, lungs and kidneys angiopathy in rats with a model of systemic autoimmune disease, carrying out comparisons of the results with extravasal morphological manifestations of the pathological process, the state of vascular endothelial function and activity of pro-inflammatory cytokines in the blood. The experiment was conducted on 40 non-linear rats. To simulate the disease animals were injected by complete Freund's adjuvant, a solution of splenic deoxyribonucleic acid cattle, mercaptopurine, methyluracilum and with food rats constantly received sulfate cadmium, lithium hydroxybutyrate and ammonium molybdate. Within two months from the start of the study animals were taken out of the experiment on the background of intraperitoneal nembutal anesthesia. Histological sections of the heart, lung and kidney tissue were stained with hematoxylin-eosin, alcian blue and by van-Gieson, becoming the PAS-reaction. In the animals with the proposed experimental model of systemic autoimmune disease observed morphological characteristics of lesions of the heart blood vessels, lungs and kidneys, the nature of the manifestations of which were interconnected with each other, which confirms the common pathogenetic angiopathy constructions at various diseases in clinical practice. In the genesis of cardiac, pulmonary and renal vessels lesions act the activation of pro-inflammatory cytokines and violation of vascular endothelial function, which has a certain practical significance. In clinical practice, not only purely systemic vasculitis, and other autoimmune diseases should be treated as angiopathy.
\end{abstract}

Keywords: autoimmune disease, experiment, rats, vessels.

\section{Introduction}

The prevalence of systemic autoimmune diseases is increasing everywhere [1-3], and the changes in heart, lungs and kidneys vessels are considered as common for them, besides the presence of various autoantibodies in serum [4-8]. The pathogenesis of such angiopathy (vasculopathy) remains studied not enough $[9,10]$, although the severity of vascular damage determines the prognosis of these diseases $[11,12]$. Animal experiments can improve the information about the role of vascular pathology in the pathogenic constructions of heart, lungs and kidneys lesions in the autoimmune systemic diseases, but the results of the following studies are still very controversial and require to be further solved [13-15]. 


\section{The purpose and objectives of the study}

To explore the nature of heart, lungs and kidneys vascular lesions in rats with autoimmune systemic disease model, to make a comparison between the results of extravascular morphological manifestations of the pathological process, vascular endothelial function and proinflammatory cytokines activity in the serum.

\section{Material and methods}

The experiment was conducted in 40 non-linear rats (10 females and 30 males) with average weight about 250 grams, according to the modified methodology which we have been proposed before [16]. To simulate the disease we used the complete Freund's adjuvant (CFA) and the solution of cattle's splenic deoxyribonucleic acid (DNA) (5 mg/kg of animal's mass) which were inserted at the root of the tail under the ether inhalation anesthesia. In two weeks mercaptopurine was administered $(50 \mathrm{mg} / \mathrm{kg}$ ) through a special tube into the stomach, and CFA and DNA $(2.5 \mathrm{mg} / \mathrm{kg})$ - in the root of the tail. On the next day during one week animals were administered methyluracilum into the stomach daily $(200 \mathrm{mg} / \mathrm{kg})$ and then repeated administration of mercaptopurine $(50 \mathrm{mg} / \mathrm{kg})$, CFA $(1.5 \mathrm{mg} / \mathrm{kg})$ and DNA $(1.5 \mathrm{mg} / \mathrm{kg})$. Then during the week animals were given methyluracilum $(100 \mathrm{mg} / \mathrm{kg})$. The rats received about 0.1 $\mathrm{mg}$ of cadmium sulfate, $500 \mathrm{mg}$ of lithium hydroxybutyrate and $0.3 \mathrm{mg}$ of ammonium molybdate with food regularly (at the rate per animal). In two months after the beginning of the study against the background of intraperitoneal nembutal anesthesia $(50 \mathrm{mg} / \mathrm{kg})$ animals were taken out of the experiment. The control group consisted of 20 intact rats.

Histological sections of heart, lungs and kidneys tissue were stained with hematoxylin-eosin, alcian blue (on glycoproteins) and with the method of van-Gieson (collagen and elastic fibers), PAS-reaction was conducted. Vascular and extravascular heart, lungs and kidneys lesions were evaluated in scores (0 to 3). There was calculated the average damage index (ADI) using a formula: $A D I=(a+2 b+3 c):(a+b+c+d)$, where $" a, b, c "-$ the number of animals in accordance with 1,2 and 3 points and " $\mathrm{d}$ " - the number of animals with the absence of this feature. The levels of proinflammatory cytokines - tumor necrosis factor $\alpha$ (TNF $\alpha$ ) and interleukin $1 \beta$ (IL1 $\beta$ ) were studied in serum by immune-enzyme analysis (reader "PR2100 Sanofi diagnostic pasteur", France), and the concentrations of endothelial vascular function - endothelin-1 (ET1) and cyclic guanosine monophosphate (cGMP) (immune-enzyme analysis (reader "PR2100 Sanofi diagnostic pasteur", France)) were associated with them.

Statistical analysis of the results of research was conducted by computer variation, correlation, regression, one-factor (ANOVA) and multiple (ANOVA/ MANOVA) analysis of variance (program "Microsoft Excel" and "Statistica-Stat-Soft", USA). The mean values, their standard deviations and error rates, coefficient of parametric Pearson correlation (r) and nonparametric Kendall $(\tau)$, regression criteria $(\mathrm{R})$, dispersion Brown-Forsythe (BF) and Wilcoxon-Rao (WR), Student's test ( $\mathrm{t}$ ) and the probability of statistical indicators (p) were assessed. The critical level of significance in testing statistical hypotheses was considered as being equal to 0.05 .

\section{Results}

In rats with the model of experimental autoimmune disease changes in the heart vessels were found in $82.5 \%$ of cases, lungs - in $32.5 \%$, kidneys - in $72.5 \%$. ADI of vascular injuries was $1,07 \pm 0,332$ points.

Morphological signs of vascular pathology of heart tissue are shown in the Fig. 1, 2.

Changes in the lungs vessels of the animals with autoimmune disease model are shown in the Fig. 3, 4.

The proliferation of glomerular capillaries endothelium was revealed in the kidneys of $82.5 \%$ of the studied rats, proliferation of arterioles endothelium - in $65.0 \%$, cellular perivascular (mainly lymphohistiocytic) infiltration of stroma - in $40.0 \%$ synechia of glomerular capsule with capillaries - in $27.5 \%$. These data are reflected in the Fig. 5, 6. 


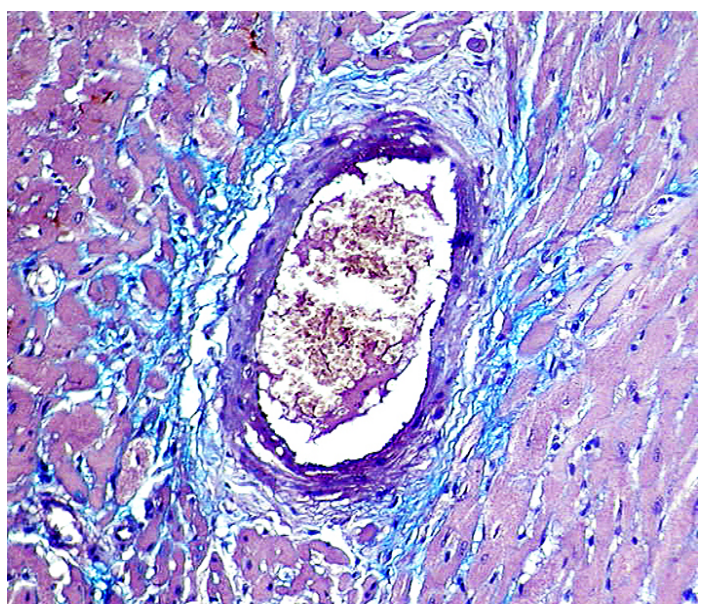

Fig. 1. Heart preparation of a rat with autoimmune systemic disease. Perivascular sclerosis (van-Gieson staining, $\times 400$ )

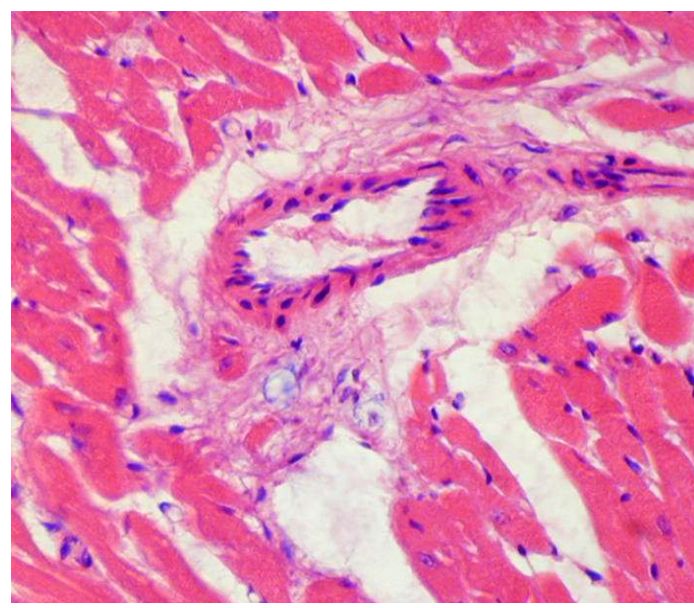

Fig. 2. Heart preparation of a rat with autoimmune systemic disease. Proliferation of arteries endothelium, perivascular sclerosis (hematoxylin and eosin staining, $\times 400$ )

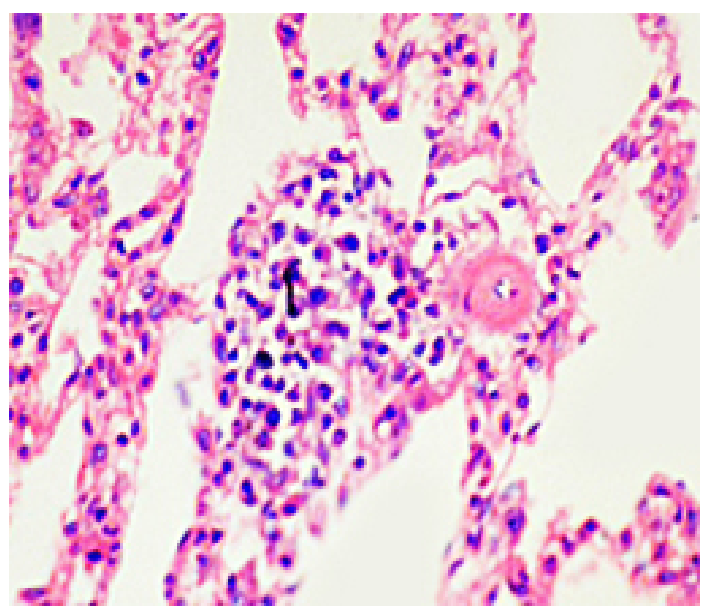

Fig. 3. Lung preparation of a rat with systemic autoimmune disease. Arterioles hyalinosis, perivascular lymphohistiocytic infiltration (hematoxylin and eosin staining, $\cdot 400$ ) 


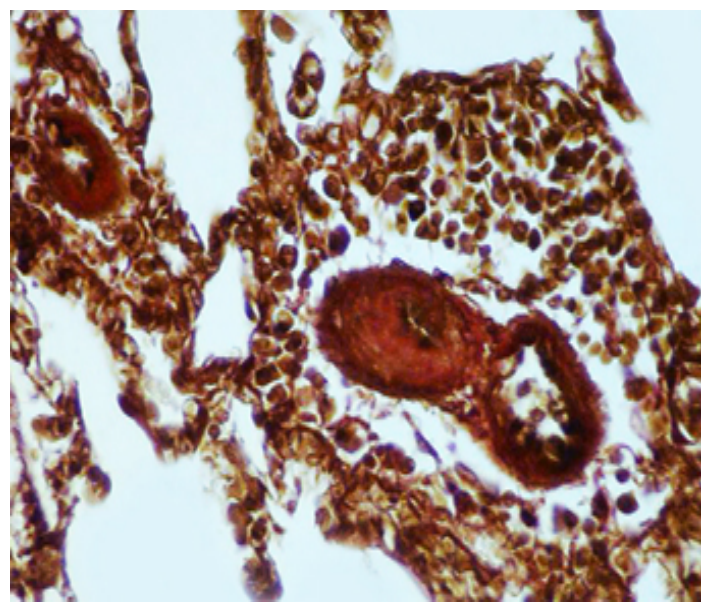

Fig. 4. Lung preparation of a rat with systemic autoimmune disease. Arterioles hyalinosis, perivascular lymphoplasmocytic infiltration (van-Gieson staining, $\cdot 400$ )

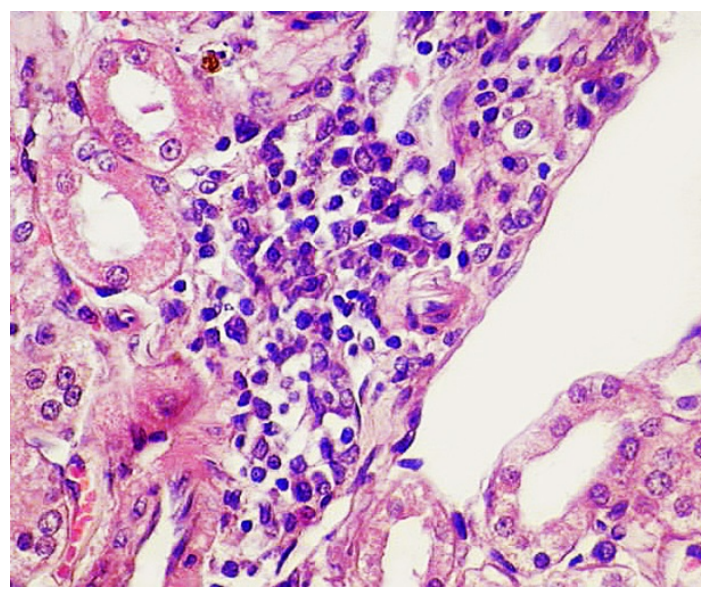

Fig. 5. Kidney preparation of a rat with autoimmune systemic disease. Adhesion of Bowman's capsule with capillaries, perivascular histiocytic infiltration (hematoxylin and eosin staining, $\cdot 400$ )

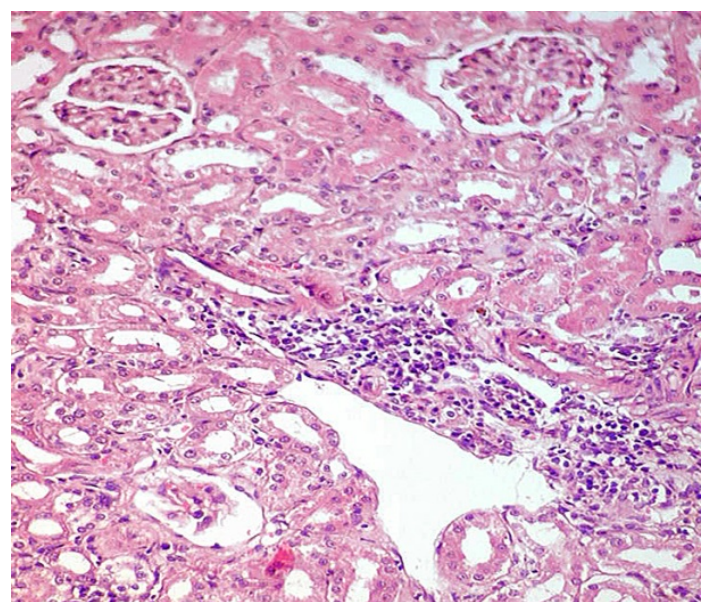

Fig. 6. Kidney preparation of a rat with autoimmune systemic disease. Perivascular lymphohistiocytic infiltration in the stroma (hematoxylin and eosin staining, 200) 
In intact animals ET1 levels in serum was $1,8 \pm 0,21 \mathrm{pg} / \mathrm{ml}, \mathrm{cGMP}-7,3 \pm 0,52 \mathrm{pmol} / \mathrm{ml}, \mathrm{TNF} \alpha-$ $21,0 \pm 0,62 \mathrm{pg} / \mathrm{ml}, \mathrm{IL} 1 \beta-14,1 \pm 0,52 \mathrm{pg} / \mathrm{ml}$. In the experimental autoimmune systemic disease the concentration of ET1 $(\mathrm{t}=9,02, \mathrm{p}<0,001)$ increased in 3.4 times, TNF $\alpha(t=5,79, \mathrm{p}<0,001)-$ in $69,6 \%$, $\operatorname{IL1} \beta(\mathrm{t}=7,88, \mathrm{p}<0,001)-$ in $78.8 \%$, but content of cGMP $(\mathrm{t}=3,16, \mathrm{p}=0,010)$ decreased in $32.2 \%$. In the experimental group of rats there are direct links between ET1 and IL1 $\beta(r=+0,597, p=0,014)$.

\section{Discussion}

In the animals with experimental model of autoimmune systemic disease are observed morphological characteristics of heart vessels lesions. Perivascular sclerosis of miocardium was detected in $3 / 4$ of the animals, venous plethora - in $52.5 \%$, vascular endothelial proliferation - in $62.5 \%$, perivascular infiltration - in $42.5 \%$, vascular sclerosis/hyalinosis - in $35.5 \%$. ADI vessels directly correlated with ADI of endocardium and valves $(r=+0,439, p=0,016)$. It is suggested the involvement of myocardial vessels in most animal models and interconnection between lesion of vessels and endocardium.

Morphological signs of vascular pathology of heart tissue are (in the decrescent order by frequency): mucoid swelling of vessels, thickening of the vascular wall, proliferation of endothelium, fibrinoid swelling, intravascular aggregation of red blood cells, intravascular lymphohistiocytic infiltration, perivascular lymphohistiocytic infiltration, lymphohistiocytic infiltration of the vascular wall, fibrinoid necrosis, neutrophil infiltration of the vascular wall, proliferation of the intima, perivascular neutrophilic infiltration, giant cell infiltration, extravasation of erythrocytes.

Changes in the lungs vessels of the animals with autoimmune disease model are characterized by the thickening of the walls, mucoid swelling, angiospasm, endothelial proliferation, perivascular lymphohistiocytic infiltration and mast cells infiltration, sclerosis/hyalinosis of vascular walls. There is abundant evidence that mast cells are active participants in events that mediate tissue damage in autoimmune disease.

According to multivariate analysis of variance Wilcoxon-Rao, integral degree changes of heart vessels significantly $(p=0,009-p<0,001)$ depends on the severity of cardiomyocytes hypertrophy $(\mathrm{WR}=10,07)$, its necrosis $(\mathrm{WR}=5,93)$, stromal edema $(\mathrm{WR}=8,28)$, lymphomacrophage its infiltration ( $\mathrm{WR}=12,25)$, and perivascular cell infiltration ( $\mathrm{WR}=5,71)$. In turn, miocardium angiopathy ADI influences on the severity of necrotic and sclerotic changes of endocardium, as Brown-Forsythe analysis indicated (correspondingly $\mathrm{BF}=8,23, \mathrm{p}=0,004$ and $\mathrm{BF}=9,77, \mathrm{p}<0,001$ ). These interconnections confirm the common pathogenetic factors for angiopathy constructions and organ's damage.

According to the single-factor analysis of variance, the ADI of endocardium and valvular lesions influence the degree of vascular endothelium proliferation $(B F=4,79, p=0,010)$. The severity of perivascular sclerosis affects the intensity of endocardium cellular infiltration $(\mathrm{BF}=3,88$, $p=0,023)$, the perivascular infiltration - endocardial necrotization $(B F=4,25, p=0,025)$, and the perivascular sclerosis - endocardial sclerosis $(B F=5,33, p=0,009)$, the cardiomyocyte necrosis - proliferation of vascular endothelium $(\mathrm{BF}=3,34, \mathrm{p}=0,035)$. The morphological characteristics of heart vascular lesions are connected to each other.

ADI of the kidneys and lungs vessels was about the same, accounting respectively $1,52 \pm 0,118$ and $1,50 \pm 0,174$ points. The degree of proliferation of heart and lungs vascular endothelium directly correlated with the kidney ADI (respectively $r=+0,521, p=0,018$ and $r=+0,538, p=0,008$ ), and the severity of the kidney vascular endothelium lesion statistically significant $(p<0,001)$ influence on the change of hearts and lungs endothelium (correspondingly $\mathrm{BF}=10,06$ and $\mathrm{BF}=8,93$ ).

According to the analysis of variance, sclerotic damage of kidneys' capillaries and arterioles depend on the degree of proliferation of heart vascular endothelium $(B F=7,47, p=0,008)$, and ADI of lungs vessels - on the endothelial proliferative changes of renal vessels $(\mathrm{BF}=4,58$, $\mathrm{p}=0.017)$. Regression analysis shows the direct influence of vascular heart endothelium degree proliferation on venous plethora $(\mathrm{R}=+4,20, \mathrm{p}=0,026)$, perivascular infiltration $(\mathrm{R}=+8,11$, $\mathrm{p}<0,001)$ and sclerosis of the heart vessels $(\mathrm{R}=+2,56, \mathrm{p}=0,040)$ are up to the condition of the pulmonary vessels, sclerosis/hyalinosis of renal arterioles $(R=+3,08, p=0,019)$ - pulmonary vascular endothelium. Kendall nonparametric analysis found direct correlation between scle- 
rotic symptoms of heart and lungs vessels $(\tau=+0,247, \mathrm{p}=0,012)$, heart and kidney $(\tau=+0,183$, $\mathrm{p}=0,038)$, lungs and kidney $(\tau=+0,204, \mathrm{p}=0.025)$. It shows the similarity of pathogenetic theories of angiopathy in autoimmune diseases.

The degree of proliferation of endothelium and vascular sclerosis influence the integrated state of vascular endothelial function in animal with model of autoimmune systemic disease as indicated the multivariate analysis of variance ANOVA/MANOVA (accordingly WR=2,36, $\mathrm{p}=0,035$ and $\mathrm{WR}=2,72, \mathrm{p}=0,013)$. Univariate Brown-Forsythe analysis shows the effect of ET1 parameters on the severity of kidney vessels endothelium proliferation $(\mathrm{BF}=4,16, \mathrm{p}=0,029)$, and the concentration of IL1 $\beta$ - on the heart vessels endothelium proliferation $(B F=12,05, p<0,001)$. In the genesis of cardiac, pulmonary and renal vessels lesions act the activation of pro-inflammatory cytokines and violation of vascular endothelial function

It should be noted that in the experimental autoimmune vascular pathology there is a complex system of protection of endothelial cells, which is directed to inhibition of pro-inflammatory cytokines, matrix metalloproteinases and C-dependent protein kinase [17]. Protection of nitrogen oxide relatively to endothelial dysfunction is negligible, and in the linear mice C57Bl/B6j, NZB/W and MRL/1 with immune disorders the additional input of autoantibodies to the basement membrane of vessels is accompanied by the generation of molecules of nitric oxide, but it does not prevent the adhesion of neutrophils to the endothelium of the capillaries and progression of the pathological process [18-20]. Bradykinin can suppress the signs of endothelial dysfunction in model of autoimmune systemic disease that enhances vasodilation [21, 22].

Our review also revealed that most animal models only recapitulate some features of human disease but the genetic heterogeneity of individual human autoimmune diseases should be considered. A single rat strain is clearly unable to encompass the heterogeneity of the human population. Thus studies should not be restricted to the identification and/or use of a "gold standard" animal model. Rather models should be investigated that best reflect human genetic heterogeneity, and/or ask specific mechanistic questions that a particular model is able to address.

Information that presented in the study required for testing of vessels (carrying sonography, capillarography, conjunctival biomicroscopy etc.) in the early stages of observation of patients with systemic autoimmune diseases for timely accomplishment of appropriate preventive measures in the context of the identified angiopathy. There is still much to be learned about how angiopathy impact autoimmunity.

\section{Conclusions}

1. In the animals with experimental model of autoimmune systemic disease are observed morphological characteristics of heart, lungs and kidneys vascular lesions, the character of which manifestations are connected to each other, as evidence by similarity of pathogenetic theories of angiopathy in autoimmune diseases in clinical practice.

2. The activation of proinflammatory cytokines and vascular endothelial function abnormality play a vital part in the genesis of cardiac, pulmonary and renal vessels lesions, which has a practical significance.

3. In clinical practice, not only systemic vasculitis (Henoch-Schonlein purpura, microscopic polyangiitis, Granulomatosis with polyangiitis (Wegener), eosinophilic granulomatosis with polyangiitis (Churg-Strauss), polyarteritis nodosa, vasculitis Behcet and Kawasaki etc.) and other autoimmune systemic rheumatic diseases (systemic lupus erythematosus, systemic scleroderma, Sjogren's disease, rheumatoid arthritis, ankylosing spondylitis etc.) should be considered as angiopathy.

4. The study of the heart, lungs and kidneys angiopathy nature in rats with a model of systemic autoimmune disease can be used for a wider investigation of pathogenesis of autoimmune diseases and for searching new approaches to the treatment of these diseases.

\section{Conflict of interest}

During the preparation of material for publication the authors did not receive fees and had no other material interest. 


\section{References}

[1] Heijnen, T., Wilmer, A., Blockmans, D., Henckaerts, L. (2015). Outcome of patients with systemic diseases admitted to the medical intensive care unit of a tertiary referral hospital: a single-centre retrospective study. Scandinavian Journal of Rheumatology, 45 (2), 146-150. doi: 10.3109/0300974 2.2015.1067329

[2] Holle, J. U. (2014). ANCA-assoziierte Vaskulitiden. Der Internist, 56 (1), 41-52. doi: 10.1007/ s00108-014-3613-8

[3] Ramos-Casals, M., Brito-Zeron, P., Kostov, B., Siso-Almirall, A., Bosch, X., Buss, D. et. al. (2015). Google-driven search for big data in autoimmune geoepidemiology: Analysis of 394,827 patients with systemic autoimmune diseases. Autoimmunity Reviews, 14 (8), 670-679. doi: 10.1016/j.autrev.2015.03.008

[4] Dey-Rao, R., Sinha, A. A. (2015). Genome-wide transcriptional profiling data from chronic cutaneous lupus erythematosus (CCLE) peripheral blood. Data in Brief, 2, 39-41. doi: 10.1016/ j.dib.2014.11.006

[5] Elshabrawy, H. A., Chen, Z., Volin, M. V., Ravella, S., Virupannavar, S., Shahrara, S. (2015). The pathogenic role of angiogenesis in rheumatoid arthritis. Angiogenesis, 18 (4), 433-448. doi: 10.1007/ s10456-015-9477-2

[6] Hoffman, G. S., Calabrese, L. H. (2014). Vasculitis: determinants of disease patterns. Nature Reviews Rheumatology, 10 (8), 454-462. doi: 10.1038/nrrheum.2014.89

[7] Kuo, C.-F., Grainge, M. J., Valdes, A. M., See, L.-C., Luo, S.-F., Yu, K.-H. et. al. (2015). Familial Aggregation of Systemic Lupus Erythematosus and Coaggregation of Autoimmune Diseases in Affected Families. JAMA Internal Medicine, 175 (9), 1518-1526. doi: 10.1001/jamainternmed.2015.3528

[8] Rawlings, C. R., Fremlin, G. A., Nash, J., Harding, K. (2015). A rheumatology perspective on cutaneous vasculitis: assessment and investigation for the non-rheumatologist. International Wound Journal, 13 (1), 17-21. doi: 10.1111/iwj.12437

[9] Petrucci, I., Samoni, S., Meola, M. Clinical Scenarios in Chronic Kidney Disease: Parenchymal Chronic Renal Diseases - Part 2. Ultrasound Imaging in Acute and Chronic Kidney Disease, 188, 98-107. doi: $10.1159 / 000445472$

[10] Wilhelmus, S., Alpers, C. E., Cook, H. T., Ferrario, F., Fogo, A. B., Haas, M. et. al. (2015). The Revisited Classification of GN in SLE at 10 Years: Time to Re-Evaluate Histopathologic Lesions. Journal of the American Society of Nephrology, 26 (12), 2938-2946. doi: 10.1681/asn.2015040384

[11] Iwasaki, S., Suzuki, A., Fujisawa, T., Sato, T., Shirai, S., Kamigaki, M. et. al. (2015). Fatal cardiac small-vessel involvement in ANCA-associated vasculitis: an autopsy case report. Cardiovascular Pathology, 24 (6), 408-410. doi: 10.1016/j.carpath.2015.07.005

[12] Marszalek, A., Skoczylas-Makowska, N., Kardymowicz, A., Manitius, J. (2010). Patient with rheumatoid arthritis and acute renal failure: a case report and review of literature. Polish Journal of Pathology, 61 (4), 229-233.

[13] Bevzenko, T. B. (2015). Renal morphological changes in experimental systemic vasculitis: relation to nephrotoxic microelements. Kidney, 3 (13), 33-36.

[14] Syniachenko, O. V., Ignatenko, G. A., Dyadyk, A. A. (2004). Changes of respiratory system in experimental models of autoimmune disease during the treatment. Ukrainian Rheumatology Journal, $18(4), 3-6$.

[15] Syniachenko, P. O., Ignatenko, G. A., Dyadyk, E. A. (2011). Endocardial and heart valves lesions at experimental systemic lupus erythematosus. Bulletin of urgen and recovery medicine, 12 (1), 102-105.

[16] Nikolenko, Yu. I., Syniachenko, O. V., Nikolenko, V. Yu, Ananeva, M. M. (1998). Method of simulation systemic autoimmune disease. Patent UA № 6G09B 23/28 30.10.98. 
[17] Ahmad, S. R., Lidington, E. A., Ohta, R., Okada, N., Robson, M. G., Davies, K. A. et. al. (2003). Decay-accelerating factor induction by tumour necrosis factor-alpha, through a phosphatidylinositol-3 kinase and protein kinase C-dependent pathway, protects murine vascular endothelial cells against complement deposition. Immunology, 110 (2), 258-268. doi: 10.1046/j.1365-2567.2003.01733.x

[18] Gilkeson, G. S., Mudgett, J. S., Seldin, M. F., Ruiz, P., Alexander, A. A., Misukonis, M. A. et. al. (1997). Clinical and Serologic Manifestations of Autoimmune Disease in MRL-lpr/lprMice Lacking Nitric Oxide Synthase Type 2. The Journal of Experimental Medicine, 186 (3), 365-373. doi: 10.1084/jem.186.3.365

[19] Heeringa, P., van Goor, H., Itoh-Lindstrom, Y., Maeda, N., Falk, R. J., Assmann, K. J. M. et. al. (2000). Lack of Endothelial Nitric Oxide Synthase Aggravates Murine Accelerated Anti-Glomerular Basement Membrane Glomerulonephritis. The American Journal of Pathology, 156 (3), 879-888. doi: 10.1016/ s0002-9440(10)64957-7

[20] Yang, Z., Ren, Y., Liu, D., Lin, F., Liang, Y. (2016). Prevalence of systemic autoimmune rheumatic diseases and clinical significance of ANA profile: data from a tertiary hospital in Shanghai, China. APMIS, 124 (9), 805-811. doi: 10.1111/apm.12564

[21] Sessa, W. C. (2009). Molecular control of blood flow and angiogenesis: role of nitric oxide. Journal of Thrombosis and Haemostasis, 7, 35-37. doi: 10.1111/j.1538-7836.2009.03424.x

[22] Gilkeson, G. S., Mudgett, J. S., Seldin, M. F., Ruiz, P., Alexander, A. A., Misukonis, M. A. et. al. (1997). Clinical and Serologic Manifestations of Autoimmune Disease in MRL-lpr/lprMice Lacking Nitric Oxide Synthase Type 2. The Journal of Experimental Medicine, 186 (3), 365-373. doi: 10.1084/jem.186.3.365 